\title{
Effect of mantle and ocean tides on the Earth's rotation rate
}

\author{
P. M. Mathews ${ }^{1}$ and S. B. Lambert ${ }^{2}$ \\ 1 Department of Theoretical Physics, University of Madras, Chennai 600025, India \\ 2 Observatoire de Paris, Département Systèmes de Référence Temps Espace (SYRTE), CNRS/UMR8630, 75014 Paris, France \\ e-mail: sebastien.lambert@obspm.fr
}

Received 7 June 2008 / Accepted 17 October 2008

\section{ABSTRACT}

\begin{abstract}
Aims. We aim to compute the rate of increase of the length of day (LOD) due to the axial component of the torque produced by the tide generating potential acting on the tidal redistribution of matter in the oceans and the solid Earth.

Methods. We use an extension of the formalism applied to precession-nutation in a previous work to the problem of length of day variations of an inelastic Earth with a fluid core and oceans. Expressions for the second order axial torque produced by the tesseral and sectorial tide-generating potentials on the tidal increments to the Earth's inertia tensor are derived and used in the axial component of the Euler-Liouville equations to arrive at the rate of increase of the LOD.

Results. The increase in the LOD, produced by the same dissipative mechanisms as in the theoretical work on which the IAU 2000 nutation model is based and in our recent computation of second order effects, is found to be at a rate of $2.35 \mathrm{~ms} / \mathrm{cy}$ due to the ocean tides, and $0.15 \mathrm{~ms} / \mathrm{cy}$ due to solid Earth tides, in reasonable agreement with estimates made by other methods.
\end{abstract}

Key words. reference systems - Earth

\section{Introduction}

The gradual slowing down of the speed of rotation of the Earth has been going on for hundreds of millions of years, as evidenced by geological records. For instance, the analysis of sedimentary cyclic rhythmites of tidal origin has revealed that about $620 \mathrm{Myr}$ ago, the length of day (LOD) was only $22 \mathrm{~h}$ (Williams 2000). Compilations by Varga et al. (1998) and Denis et al. (2002), of the estimates by various authors for the LOD over geological time, show that the LOD increased over the last 0.5 Gyr, at a rate of about $1.79 \pm 0.18 \mathrm{~ms}$ per century (ms/cy), wherein the non-tidal acceleration attributed to the Earth's viscous postglacial rebound is included.

The Earth's slowing down is explained as the effect of the axial component of the torque exerted by the tidal potential of the Moon on the density perturbation produced in the Earth by the deformational action of the tidal potential itself. This deformational response is delayed relative to the forcing potential because of the dissipative mechanisms present in the oceans and in the solid Earth; consequently the torque retards the Earth's rotation, and the reciprocal torque on the Moon causes an energy transfer to the Moon, resulting in a gradual increase in the Eath-Moon distance. During a large part of the 20th century, most of the dissipative mechanism in the oceans was thought to occur on shallow sea bottoms (Jeffreys 1929). Tidal dissipation in the deep ocean was considered to be negligible (Lamb 1945). Recent works that take advantage of TOPEX/Poseidon measurements of the ocean surface showed, however, that though most of the friction does occur in the shallow seas, a non-negligible part (up to $30 \%$, according to Egbert \& Ray 2001) comes from the deep ocean, generally near areas of rough ocean bottom topography (e.g., the Mid-Atlantic or western Pacific ridges, see Jayne \& St. Laurent 2001).

Lengthening of the day can be calculated from the concomitant effect on the lunar orbit (see, e.g., Williams 1994; Chapront et al. 2002), or directly estimated from models of ocean and solid Earth tidal responses. Among various studies, Lambeck (1977) mentions an increase in the length of day of $2.7 \mathrm{~ms} / \mathrm{cy}$ that includes a significant non-tidal acceleration of $0.7 \pm 0.4 \mathrm{~ms} / \mathrm{cy}$, leaving some $2 \mathrm{~ms} / \mathrm{cy}$ for the tidal dissipation in both the oceans and the mantle. Ray et al. (1999) calculated the lunar and solar torques on the ocean tides using Geosat and TOPEX/Poseidon altimetry data. They obtained a rate of change of the LOD of $2.28 \mathrm{~ms} / \mathrm{cy}$.

In an earlier paper (Lambert \& Mathews 2006), a formalism was developed to compute the equatorial components of the second order torque due to external gravitational action on the tidal redistribution of matter in the Earth, and hence to compute the resulting second-order corrections to the nutation. At that time we ignored the equations relating to the third (axial) component of the Earth's rotation vector which govern the variations in the Earth's rotation speed. In the present paper, we extend our previous computations to the axial component, and compute the expressions for the axial torques produced by sectorial and tesseral tidal potentials acting on solid Earth and ocean tidal deformations, and go on to calculate the resultant rate of increase of the LOD. Such an approach differs from that of Ray et al. since we start from more general equations. Moreover, we do not limit our study to ocean tides but include also mantle tides using Earth parameters derived from the IAU 2000 nutation model (Mathews et al. 2002, hereafter referred to as MHB). 


\section{Earth's rotation rate variations}

The variations in the Earth's rotation rate are governed by the third component of the vector equation of angular momentum conservation. In a frame attached to the rotating Earth, it is

$\frac{\mathrm{d} H_{3}}{\mathrm{~d} t}=\Gamma_{3}-[\mathbf{\Omega} \times \boldsymbol{H}]_{3}$,

where $H_{3}$ is third component of the angular momentum $\boldsymbol{H}$ of the Earth, $\boldsymbol{\Omega}=\Omega_{0}\left(m_{1}, m_{2}, 1+m_{3}\right)$ is the instantaneous angular velocity vector, and $\Gamma_{3}$ is the third component of the external torque. The variation of the rotation rate is $\dot{\Omega}_{3}=\Omega_{0} \dot{m}_{3} ; m_{3}$ enters the equation primarily through the expression for $\mathrm{H}_{3}$ which is given, to the second order in small quantities, by

$$
\begin{aligned}
\Omega_{0}^{-1} H_{3} & =\left(C+c_{33}\right)\left(1+m_{3}\right)+c_{13} m_{1}+c_{23} m_{2}+\left(C_{\mathrm{f}}+c_{33, \mathrm{f}}\right) m_{3, \mathrm{f}}+c_{13, \mathrm{f}} m_{1, \mathrm{f}}+c_{23, \mathrm{f}} m_{2, \mathrm{f}} \\
& =\Omega_{0}^{-1}\left(H_{3}^{(0)}+H_{3}^{(1)}+H_{3}^{(2)}\right),
\end{aligned}
$$

where

$\Omega_{0}^{-1} H_{3}^{(0)}=C$,

$\Omega_{0}^{-1} H_{3}^{(1)}=C m_{3}+c_{33}+C_{\mathrm{f}} m_{3, \mathrm{f}}$,

$\Omega_{0}^{-1} H_{3}^{(2)}=c_{33} m_{3}+c_{33, \mathrm{f}} m_{3, \mathrm{f}}+\frac{1}{2}\left(\tilde{c}_{3} \tilde{m}^{*}+\tilde{c}_{3}^{*} \tilde{m}\right)+\frac{1}{2}\left(\tilde{c}_{3, \mathrm{f}} \tilde{m}_{\mathrm{f}}^{*}+\tilde{c}_{3, \mathrm{f}}^{*} \tilde{m}_{\mathrm{f}}\right)$.

In these expressions, $C$ is the dominant time independent part of the Earth's moment of inertia (including the contribution from the zero frequency part of the tidal potential) about the third axis. The $c_{i j}$ are the elements of the time dependent part of the inertia tensor of the whole Earth; and $\tilde{m}$ and $\tilde{c}_{3}$, which are both functions of time, are defined by

$\tilde{m}=m_{1}+\mathrm{i} m_{2}, \quad \tilde{c}_{3}=c_{13}+\mathrm{i} c_{23}$.

Similar quantities carrying the subscript $\mathrm{f}$ pertain to the fluid core region.

The third component of $\boldsymbol{\Omega} \times \boldsymbol{H}$ is of the second order; it is given by

$$
\begin{aligned}
\Omega_{0}^{-1}[\mathbf{\Omega} \times \boldsymbol{H}]_{3} & =m_{1} H_{2}-m_{2} H_{1} \\
& =m_{1}\left(B m_{2}+c_{23}\right)+m_{1} B_{\mathrm{f}} m_{2, \mathrm{f}}-m_{2}\left(A m_{1}+c_{13}\right)-m_{2} A_{\mathrm{f}} m_{1, \mathrm{f}},
\end{aligned}
$$

where $A$ and $B$ are the constant parts of the first two diagonal elements of the inertia tensor. Setting $B=A$ and $B_{\mathrm{f}}=A_{\mathrm{f}}$ in these second order terms (i.e., neglecting triaxiality, which is of the order of $10^{-5}$ ), we simplify the above expression to

$$
\begin{aligned}
\Omega_{0}^{-1}[\mathbf{\Omega} \times \boldsymbol{H}]_{3} & =\left(m_{1} H_{2}-m_{2} H_{3}\right)=A\left(m_{1} c_{23}-m_{2} c_{13}\right)+A_{\mathrm{f}}\left(m_{1} m_{2, \mathrm{f}}-m_{2} m_{1, \mathrm{f}}\right) \\
& =\frac{1}{2 \mathrm{i}}\left[\left(\tilde{m}^{*} \tilde{c}_{3}-\tilde{m} \tilde{c}_{3}^{*}\right)+A_{\mathrm{f}}\left(\tilde{m}^{*} \tilde{m}_{\mathrm{f}}-\tilde{m} \tilde{m}_{\mathrm{f}}^{*}\right)\right] .
\end{aligned}
$$

Now, what we are seeking to determine is the secular variation of $m_{3}$. In the long term, the mantle and the core rotate together, so the secular part of the differential rotation $m_{3, \mathrm{f}}$ between the two regions vanishes, and hence that of $\dot{m}_{3, \mathrm{f}}$ too. Therefore the constant (zero frequency) part of $\Omega_{0}^{-1} \mathrm{~d} H_{3}^{(1)} / \mathrm{d} t$ reduces to

$\Omega_{0}^{-1}\left(\frac{\mathrm{d} H_{3}^{(1)}}{\mathrm{d} t}\right)_{0}=C \dot{m}_{3}(0)+\dot{c}_{33}(0)$,

wherein the subscript 0 as well as the argument (0) stand for "zero frequency". The torque equation (1) may now be written as

$C \dot{m}_{3}(0)=-\dot{c}_{33}(0)+\Omega_{0}^{-1}\left(\Gamma_{3}-\frac{\mathrm{d} H_{3}^{(2)}}{\mathrm{d} t}-[\boldsymbol{\Omega} \times \boldsymbol{H}]_{3}\right)_{0}$.

Thus, what we need to do is to determine the zero-frequency components appearing on the right hand side of the above equation. In doing so, we need to take note of the following points. Firstly, $c_{11}, c_{22}, c_{33}$ are simply the perturbations $\Delta A, \Delta B, \Delta C$ of the principal moments of inertia, and it is known that their sum is zero for inertia tensor perturbations associated with deformations having any arbitrary spherical harmonic structure. Hence $c_{33}=\Delta C=-2 \Delta \bar{A}$ where $\bar{A}=\frac{1}{2}(A+B)$, and consequently,

$c_{33}=\frac{2}{3} \Delta(C-\bar{A})=\frac{2}{3} M_{\mathrm{E}} a^{2} \Delta J_{2}, \quad$ and $\quad \dot{c}_{33}=\frac{2}{3} M_{\mathrm{E}} a^{2} \dot{J}_{2}$,

where $M_{\mathrm{E}}$ and $a$ are the mass and the equatorial radius of the Earth, respectively.

Thus, knowledge of the constant part of the $J_{2}$ rate is what is needed in obtaining the first term on the right hand side of Eq. (13).

We note that the contributions from the last two terms in (13), being of the form $m_{i} m_{j}$ or $c_{i j} m_{k}$ as may be seen from Eqs. (6) and (9), are expected to be of much smaller magnitude than the torque $\Gamma_{3}$, and hence negligible for our purposes. Nevertheless, if an evaluation of these terms were to be made, one should keep in mind that the behaviour of the axial rotation of the fluid core when the Earth is subject to tidal excitations at non-zero frequencies is quite different from that in a steadily rotating Earth: while the core rotates along with the mantle (i.e., the differential rotation $m_{3, \mathrm{f}}$ of the core with respect to the mantle is zero) under steady rotation, the core is virtually decoupled from the mantle and its axial rotation in space remains unaffected, i.e., $m_{3}+m_{3, \mathrm{f}}=0$, for excitation at tidal frequencies. This observation would be of relevance if the term involving $m_{3, \mathrm{f}}$ in $H_{3}^{(2)}$ were to be considered. However, as observed above, the last two terms in (13) are too small to be of interest in the present context. 


\section{Tesseral and sectorial potentials acting on tidal deformations: contributions to $\boldsymbol{\Gamma}_{\mathbf{3}}$}

We go on now to derive expressions for the contributions to the torque term $\Gamma_{3}$ in (13) from the action of the tesseral and sectorial potentials.

In the tesseral case,

$\boldsymbol{\Gamma}^{\mathrm{T}}=\int \rho \boldsymbol{r} \times\left(-\nabla V_{21}(\boldsymbol{r}, t)\right) \mathrm{d}^{3} r$

(the superscript T is for "tesseral") with $V_{21}=-\Omega_{0}^{2}\left[\phi_{21}^{\mathrm{re}} x z+\phi_{21}^{\mathrm{im}} y z\right]$, where $\phi_{21}^{\mathrm{re}}$ and $\phi_{21}^{\mathrm{im}}$ are functions of time. Since

$-\nabla V_{21}=\Omega_{0}^{2}\left(\begin{array}{c}\phi_{21}^{\mathrm{re}} z \\ \phi_{21}^{\mathrm{im}} z \\ \phi_{21}^{\mathrm{re}} x+\phi_{21}^{\mathrm{im}} y\end{array}\right)$,

we see that $\Gamma_{3}^{\mathrm{T}}$ is the integral of $\Omega_{0}^{2} \rho\left[x \phi_{21}^{\mathrm{im}} z-y \phi_{21}^{\mathrm{re}} z\right]$. It reduces to

$\Gamma_{3}^{\mathrm{T}}=\Omega_{0}^{2}\left[-\phi_{21}^{\mathrm{im}} c_{13}+\phi_{21}^{\mathrm{re}} c_{23}\right]=\frac{1}{2} \mathrm{i} \Omega_{0}^{2}\left[\tilde{c}_{3}^{*} \tilde{\phi}^{\mathrm{T}}-\tilde{c}_{3} \tilde{\phi}^{\mathrm{T} *}\right]$,

where

$\tilde{\phi}^{\mathrm{T}}=\phi_{21}^{\mathrm{re}}+\mathrm{i} \phi_{21}^{\mathrm{im}}$.

In the sectorial case, $V_{22}=-\Omega_{0}^{2}\left[\phi_{22}^{\mathrm{re}}\left(x^{2}-y^{2}\right)+2 \phi_{22}^{\mathrm{im}} x y\right]$ replaces $V_{21}$ in the integral (15). Then

$-\nabla V_{22}=2 \Omega_{0}^{2}\left(\begin{array}{c}\phi_{22}^{\mathrm{re}} x+\phi_{22}^{\mathrm{im}} y \\ \phi_{22}^{\mathrm{re}}(-y)+\phi_{22}^{\mathrm{im}} x \\ 0\end{array}\right)$,

and $\left[\boldsymbol{r} \times\left(-\nabla V_{22}\right)\right]_{3}=-2 \phi_{22}^{\mathrm{re}} x y+\phi_{22}^{\mathrm{im}}\left(x^{2}-y^{2}\right)$. Hence, evaluation of the integral of the form (15) with $V_{21}$ replaced by $V_{22}$ yields

$\Gamma_{3}^{\mathrm{S}}=4 \Omega_{0}^{2}\left[\left(c_{12} \phi_{22}^{\mathrm{re}}-c_{11}^{\mathrm{S}} \phi_{22}^{\mathrm{im}}\right]=2 \mathrm{i} \Omega_{0}^{2}\left[c_{+}^{\mathrm{S} *} \tilde{\phi}^{\mathrm{S}}-c_{+}^{\mathrm{S}} \tilde{\phi}^{\mathrm{S} *}\right]\right.$,

$c_{+}^{\mathrm{S}}=c_{11}^{\mathrm{S}}+\mathrm{i} c_{12}, \quad \tilde{\phi}^{\mathrm{S}}=\phi_{22}^{\mathrm{re}}+\mathrm{i} \phi_{22}^{\mathrm{im}}$,

where the superscript S stands for "sectorial".

In the above, we have used the fact that only the sectorial deformations contribute to the difference $c_{11}-c_{22}$, which reduces then to $2 c_{11}^{\mathrm{S}}$ because $c_{22}^{\mathrm{S}}=-c_{11}^{\mathrm{S}}$.

The torque $\Gamma_{3}$ in Eqs. (1) and (13) is the sum of the expressions (17) and (20).

\section{Spectral expansions}

The spectral expansions of $\tilde{\phi}^{\mathrm{T}}(t)$ and $\tilde{\phi}^{\mathrm{S}}(t)$ are as follows:

$\tilde{\phi}^{\mathrm{T}}(t)=\sum_{\omega} \tilde{\phi}_{\omega}^{\mathrm{T}}(t)=\sum_{\omega} \tilde{\phi}^{\mathrm{T}}(\omega) \mathrm{e}^{-\mathrm{i}\left(\Theta_{\omega}(t)-\pi / 2\right)}=\mathrm{i} \sum_{\omega} \tilde{\phi}^{\mathrm{T}}(\omega) \mathrm{e}^{-\mathrm{i} \Theta_{\omega}(t)}$,

$\tilde{\phi}^{\mathrm{S}}(t)=\sum_{\omega} \tilde{\phi}^{\mathrm{S}}(\omega) \mathrm{e}^{-\mathrm{i} \Theta_{\omega}(t)}$,

where $\Theta_{\omega}(t)$ is the argument of the tidal constituent of frequency $\omega$.

Now, the generic terms in the Cartwright \& Tayler (1971) spectral expansions of the tidal potential $V_{21}$ and $V_{22}$, at any point within the Earth at geocentric longitude $\lambda$ and colatitude $\theta$, are $g N_{21} H_{\omega}^{21} P_{21}(\cos \theta) \sin \left(\Theta_{\omega}(t)+\lambda\right)$ and $g N_{22} H_{\omega}^{22} P_{22}(\cos \theta) \sin \left(\Theta_{\omega}(t)+\right.$ $2 \lambda$ ), respectively, (with $N_{21}=-(5 / 24 \pi)^{1 / 2}$ and $\left.N_{22}=(5 / 96 \pi)^{1 / 2}\right)$. Equating these spectral terms to the generic terms in the spectral expansions obtained by substituting (22) and (23) into the expressions for $V_{21}$ and $V_{22}$ employed in the last section, one finds the following relations connecting the dimensionless amplitudes $\tilde{\phi}^{\mathrm{T}}$ and $\tilde{\phi}^{\mathrm{S}}$ to the Cartwright \& Tayler amplitudes $H_{\omega}^{21}$ and $H_{\omega}^{22}$, respectively:

$$
\begin{aligned}
& \tilde{\phi}^{\mathrm{T}}(\omega)=\frac{3 g N_{21} H_{\omega}^{21}}{\Omega_{0}^{2} a^{2}}=-\left(\frac{15}{8 \pi}\right)^{1 / 2} \frac{g H_{\omega}^{21}}{\Omega_{0}^{2} a^{2}}, \\
& \tilde{\phi}^{\mathrm{S}}(\omega)=\frac{3 g N_{22} H_{\omega}^{22}}{\Omega_{0}^{2} a^{2}}=\left(\frac{15}{32 \pi}\right)^{1 / 2} \frac{g H_{\omega}^{22}}{\Omega_{0}^{2} a^{2}} .
\end{aligned}
$$


The increments $c_{i j}$ appearing in the expressions (17) and (20), which are also functions of time, are related through McCullagh's theorem to the corresponding increments $\Delta C_{l m}$ and $\Delta S_{l m}$ in the geopotential coefficients:

$\tilde{c}_{3} \equiv c_{13}+\mathrm{i} c_{23}=-M_{\mathrm{E}} a^{2}\left(\Delta C_{21}+\mathrm{i} \Delta S_{21}\right)$,

$c_{+}^{\mathrm{S}} \equiv c_{11}^{\mathrm{S}}+\mathrm{i} c_{12}=-2 M_{\mathrm{E}} a^{2}\left(\Delta C_{22}+\mathrm{i} \Delta S_{22}\right)$.

When these increments are due to the redistribution of matter associated with the ocean tides and the solid Earth deformations caused by them, the spectral expansions of $\tilde{c}_{3}$ and $c_{+}^{\mathrm{S}}$ may be obtained by combining Eqs. (3) and (2b) of Chapter 6 of the IERS Conventions 2003 with Eq. (13) of the same Chapter. One finds that

$\tilde{c}_{3}^{\mathrm{OT}}=-M_{\mathrm{E}} a^{2} L_{2} \sum_{\omega}\left[\left(C_{\omega 21}^{+}+\mathrm{i} S_{\omega 21}^{+}\right) \mathrm{e}^{-\mathrm{i} \Theta_{\omega}}+\left(C_{\omega 21}^{-}-\mathrm{i} S_{\omega 21}^{-}\right) \mathrm{e}^{\mathrm{i} \Theta_{\omega}}\right]$,

$c_{+}^{\mathrm{S}, \mathrm{OT}}=-2 M_{\mathrm{E}} a^{2} L_{2} \sum_{\omega}\left[\left(C_{\omega 22}^{+}+\mathrm{i} S_{\omega 22}^{+}\right) \mathrm{e}^{-\mathrm{i} \Theta_{\omega}}+\left(C_{\omega 22}^{-}-\mathrm{i} S_{\omega 22}^{-}\right) \mathrm{e}^{\mathrm{i} \Theta_{\omega}}\right]$,

wherein the superscripts OT label the ocean tidal part, and

$L_{2}=\frac{4 \pi G \rho_{w}}{5 g}\left(1+k_{2}^{\prime}\right), \quad M_{\mathrm{E}} a^{2} L_{2}=\frac{4 \pi \rho_{w} a^{4}}{5}\left(1+k_{2}^{\prime}\right)$,

where $\rho_{w}$ is the density of sea water and $k_{2}^{\prime}$ is the load Love number.

\section{Evaluation of the torques}

Ocean tide contributions to the axial torques (17) and (20) make up the major part of these torques, so we consider these contributions first. Each of the two expressions is a sum of two mutually complex conjugate parts, and each part is a product of two functions of time. One function represents the tidal deformation itself, and the other represents the external tidal excitation (or potential). The spectral frequencies present in the two functions are labelled by $\omega_{1}$ and $\omega_{2}$, respectively. On using the expansions (22) and (28), one can see that

$\Gamma_{3}^{\mathrm{T}, \text { OT }}=M_{\mathrm{E}} a^{2} L_{2} \Omega_{0}^{2} \operatorname{Re} \sum_{\omega_{1}} \sum_{\omega_{2}}\left[\left(C_{\omega_{1} 21}^{+}-\mathrm{i} S_{\omega_{1} 21}^{+}\right) \mathrm{e}^{\mathrm{i} \Theta_{\omega_{1}}}+\left(C_{\omega_{1} 21}^{-}+\mathrm{i} S_{\omega_{1} 21}^{-}\right) \mathrm{e}^{-\mathrm{i} \Theta_{\omega_{1}}}\right] \tilde{\phi}^{\mathrm{T}}\left(\omega_{2}\right) \mathrm{e}^{-\mathrm{i} \Theta_{\omega_{2}}}$.

Similary, one finds with the use of (25) and (29) that

$\Gamma_{3}^{\mathrm{S}, \text { OT }}=-4 M_{\mathrm{E}} a^{2} L_{2} \Omega_{0}^{2} \operatorname{Re} \sum_{\omega_{1}} \sum_{\omega_{2}} \mathrm{i}\left[\left(C_{\omega_{1} 22}^{+}-\mathrm{i} S_{\omega_{1} 22}^{+}\right) \mathrm{e}^{\mathrm{i} \Theta_{\omega_{1}}}+\left(C_{\omega_{1} 22}^{-}+\mathrm{i} S_{\omega_{1} 22}^{-}\right) \mathrm{e}^{-\mathrm{i} \Theta_{\omega_{1}}}\right] \tilde{\phi}^{\mathrm{S}}\left(\omega_{2}\right) \mathrm{e}^{-\mathrm{i} \Theta_{\omega_{2}}}$

We are interested primarily in the zero frequency part of the torques, which is responsible for the part of the rotation rate variation that is linear in time. Since the tidal frequencies are non-negative, the zero frequency terms arise only from the first part of each expression, having the time dependence $\mathrm{e}^{\mathrm{i}\left(\Theta_{\omega_{1}}-\Theta_{\omega_{2}}\right)}$ and that too, only for $\omega_{2}=\omega_{1}=\omega$, say. The constant part of the total axial torque $\Gamma_{3}^{\mathrm{T}}+\Gamma_{3}^{\mathrm{S}}$ is thus

$\Gamma_{3}^{(0), \text { От }}=M_{\mathrm{E}} a^{2} L_{2} \Omega_{0}^{2} \sum_{\omega}\left[C_{\omega 21}^{+} \tilde{\phi}^{\mathrm{T}}(\omega)-4 S_{\omega 22}^{+} \tilde{\phi}^{\mathrm{S}}(\omega)\right]$,

where the superscript (0) on $\Gamma_{3}$ is simply to indicate that it is the zero frequency component. One may susbstitute for $\tilde{\phi}^{\mathrm{T}}(\omega)$ and $\tilde{\phi}^{\mathrm{S}}(\omega)$ from Eqs. (24) and (25) in displaying the dependence of $\Gamma_{3}$ on the basic parameters.

Is the sign of the torque generated by each tidal component negative, as one would expect? In looking at this question, we note from (24) that $\tilde{\phi}^{\mathrm{T}}(\omega)$ is $\left(-H_{\omega}^{21}\right)$ times a positive constant, and hence that the first term in (33) will be negative provided that $C_{\omega 21}^{+} H_{\omega}^{21}$ is positive. On the other hand, (25) shows that $\tilde{\phi}^{\mathrm{S}}$ is a positive constant times $H_{\omega}^{22}$ and hence a negative contribution will arise from the second term too if $S_{\omega 22}^{+} H_{\omega}^{22}$ is positive. An examination of the Cartwright \& Tayler tables for the $H_{\omega}^{2 m}$ and the CSR4 tables for the ocean tide heights $C_{\omega 2 m}^{ \pm}$and $S_{\omega 2 m}^{ \pm}$shows that both the above conditions on the signs are satisfied. Therefore our physical expectation is indeed borne out.

Consider next the contribution to $\Gamma_{3}$ from anelastic dissipation in the solid Earth tides. It is most convenient to incorporate this effect into the integral (17) by expressing the solid Earth parts $\tilde{c}_{3}^{\mathrm{SE}}$ and $c_{+}^{\mathrm{SSE}}$ of $\tilde{c}_{3}$ and $\tilde{c}_{+}^{\mathrm{S}}$ in terms of the relevant compliances $\kappa$ and $\xi$ (see, for instance, Sasao et al. 1980):

$\tilde{c}_{3}^{\mathrm{SE}}=-A\left[\kappa\left(\tilde{\phi}^{\mathrm{T}}-\tilde{m}\right)-\xi \tilde{m}_{\mathrm{f}}\right]$.

The corresponding expression for $c_{+}^{\mathrm{S} S \mathrm{SE}}$ is to be introduced into (20):

$\tilde{c}_{+}^{\mathrm{S}, \mathrm{SE}}=-2 A \kappa \tilde{\phi}^{\mathrm{S}}$.

The value of $\kappa$ for the tesseral tides is taken from Mathews et al. (2002); the estimate of 0.0010340 shown in Table 2 of that paper for $\kappa$ of the elastic Earth, plus the increment of $(1.26+\mathrm{i} 0.53) \times 10^{-5}$ arising from anelasticity yields the complex value 
Table 1. Contribution to the angular acceleration $\Omega_{0} \dot{m}_{3}$ and to the LOD rate from the action of tidal potentials on ocean and solid Earth tides.

\begin{tabular}{llrr}
\hline \hline & Tidal potentials & $\begin{array}{r}\text { Angular acceleration } \\
\text { "/cy }\end{array}$ & $\begin{array}{r}\text { LOD rate } \\
\mathrm{ms} / \mathrm{cy}\end{array}$ \\
\hline Solid Earth & Sectorial & -65 & 0.12 \\
- & Tesseral & -15 & 0.03 \\
- & Total & -80 & 0.15 \\
\hline Ocean & Sectorial & -1084 & 1.98 \\
- & Tesseral & -205 & 0.37 \\
- & Total & -1369 & 2.35 \\
\hline Total Solid Earth + Ocean & -1449 & 2.50 \\
\hline
\end{tabular}

$(1.0466+\mathrm{i} 0.0053) \times 10^{-3}$ for the $\kappa^{\mathrm{T}}$ of the dissipative Earth, excluding the ocean tide effects. The value of $\xi$, including the anelasticity contribution, is $(2.247+\mathrm{i} 0.986) \times 10^{-4}$. The values of $\tilde{m}$ and $\tilde{m}_{\mathrm{f}}$ for the relevant frequencies in the diurnal band are taken from the calculations on the MHB theory; actually the contributions from the $\tilde{m}$ term in $\tilde{c}_{3}$ are negligible while those from the $\tilde{m}_{\mathrm{f}}$ term are significant for just a few frequencies near the nearly diurnal free wobble (NDFW) resonance.

For excitations at the semidiurnal frequencies of the sectorial potential, the value of $\kappa$ is slightly different $\left(\kappa^{\mathrm{S}}=(1.0538+\right.$ i0.0046) $\times 10^{-3}$ ), partly because of the difference in the anelasticity contribution between the semidiurnal and diurnal frequencies and partly because the ellipticity of the Earth increments $\kappa$ by slightly different amounts in the different frequency bands.

\section{Numerical results}

Contributions from solid Earth and ocean tides to the increments of inertia appearing in the torque expressions (17) and (20) have been evaluated. They were obtained from the solution of the dynamical equations for the rotation of an inelastic Earth with a fluid core, as explained in Sect. 4 of Lambert \& Mathews (2006). The ocean tide heights have been taken from the CSR4 tables (which are an extension of the CSR3 release, see Eanes \& Bettadpur 1996). The CSR4 tables do not provide error estimates on the tidal heights. However, Ray et al. mention an error of roughly $1 \%$ on the height of the M2 tidal constituent of the EGM96S model (Lemoine et al. 1998). Moreover, one should address the uncertainty in the value of $\kappa$. This value is available from two sources. First, from calculations for the relevant frequency band (semi-diurnal, diurnal, and low-frequency) using Earth models like PREM (Dziewonski \& Anderson 1981) based on seismological data. The uncertainties in the Earth models are generally believed to be around $1 \%$, leading to the same uncertainty in the Love number $k_{2}$ to which the compliance $\kappa$ is essentially equivalent. Second, estimations from the fit of nutation theory results to VLBI data: the MHB estimate for $\kappa$ comes with an uncertainty of about $0.3 \%$. To be conservative, we will take $1 \%$ as a safe estimate of the error.

From Eq. (13), one gets the angular acceleration $\Omega_{0} \dot{m}_{3}$, in units of " $/ \mathrm{cy}^{2}$, which can be turned into an equivalent LOD rate (in units of $\mathrm{ms} / \mathrm{cy}$ ) following

$\frac{\mathrm{dLOD}}{\mathrm{d} t}=-\Omega_{0} \dot{m}_{3} \times \mathrm{LOD}$

where the nominal value LOD is equal to $1 / 36525$ century.

The secular variations of the length of day produced by sectorial and tesseral tidal potentials are reported in Table 1. Ray et al., using various oceanic models released between 1991 and 1998, found a value of $2.28 \mathrm{~ms} / \mathrm{cy}$ for the ocean contribution including the atmospheric tides. Adding the contribution of $-0.1 \mathrm{~ms} / \mathrm{cy}$ from the atmosphere (equivalent to $55^{\prime \prime} / \mathrm{cy}^{2}$ in the rotation rate) to the oceanic contribution of $2.35 \mathrm{~ms} / \mathrm{cy}$, we get the approximate value of $2.25 \mathrm{~ms} / \mathrm{cy}$ which differs from that of Ray et al. by less than $0.03 \mathrm{~ms} / \mathrm{cy}$. The contribution due to solid Earth tides is smaller by a factor of 20, which represents about $6 \%$ of the total. The contribution of the oceans and the solid Earth tides leads to $2.50 \mathrm{~ms} / \mathrm{cy}$. The full contribution of the mantle, the oceans and the atmosphere amounts to $2.40 \mathrm{~ms} / \mathrm{cy}$. Finally, adding $-0.55 \mathrm{~ms} / \mathrm{cy}$ due to the non-tidal acceleration (Yoder et al. 1983), the total rate of change in the LOD amounts to $1.85 \mathrm{~ms} / \mathrm{cy}$. All these values have a standard error of $1 \mathrm{~ms} / \mathrm{cy}$.

In addition to secular variation of the LOD, periodic variations arise too from the interaction of the solid Earth and ocean tides with the tesseral and sectorial potentials. They remain below the current observational accuracy of the length of day. The largest contributions have amplitudes of $0.35 \mu$ s at the 18.6-year period and $0.24 \mu$ s at a semi-annual period.

In closing, we wish to note that the result obtained in this work for the tidally induced rate of increase of LOD as well as that for the obliquity rate calculated in an earlier work (Lambert \& Mathews 2008), both based on the same theoretical formulation and the same dissipative mechanisms as were employed in the MHB nutation theory (Mathews et al. 2002), are close to the results of other reliable calculations that have appeared in the recent literature like Ray et al. (1999).

\section{References}

Cartwright, D. E., \& Tayler, R. J. 1971, Geophys. J. R. Astron. Soc., 23, 45

Chapront, J., Chapront-Touzé, M., \& Francou, G. 2002, A\&A, 387, 700

Denis, C., Rybicki, K. R., \& Varga, P. 2002, presented at the 90th Journées Luxembourgeoises de Géodynamique, 4-6 November 2002

Dziewonski, A. M., \& Anderson, D. L. 1981, PEPI, 25, 297

Eanes, R., \& Bettadpur, S. 1996, Center for Space Research, Univ. Texas at Austin, Techn. Memorandum, CSR-TM-96-05

Egbert, G. D., \& Ray, R. D. 2000, Nature, 405, 775

Jayne, S. R., \& St. Laurent, L. C. 2001, Geophys. Res. Lett., 28, 811 
Jeffreys, H. 1920, Phil. Trans. R. Soc. London A, 221, 239

Lamb, H. 1945, Hydrodynamics, 6th edn. (Dover Publications)

Lambeck, K. 1977, Phil. Trans. R. Soc. London A, 287, 545

Lambert, S. B., \& Mathews, P. M. 2006, A\&A, 453, 363

Lambert, S. B., \& Mathews, P. M. 2008, A\&A, 481, 883

Lemoine, F. G., Kenyon, S. C., Factor, J. K., et al. 1998, NASA, TP-1998-206861

Mathews, P. M., Herring, T. A., \& Buffett, B. A. 2002, J. Geophys. Res., 107, 10.1029/2001JB000390

Ray, R. D., Bills, B. G., \& Chao, B. F. 1999, J. Geophys. Res., 104, 1653

Sasao, T., Okubo, S., \& Saito, M. 1980, in Proc. IAU Symp., 78, ed. E. P. Federov et al. (Hingham, MA: D. Reidel), 165

Varga, P., Denis, C., \& Varga, T. 1998, J. Geodyn., 25, 61

Williams, G. E. 2000, Rev. Geophys., 38, 37

Williams, J. G. 1994, AJ, 108, 711

Yoder, C. F., Williams, J. G., Dickey, J. O., et al. 1983, Nature, 303, 757 